\title{
Trichinosis complicated with hepatitis and myopericarditis
}

\author{
Boulus M, Saad E, Budman D, Lisitsin S, Barhoum m, Sbeit W and Assy N* \\ Department of Internal Medicine A, Galilee medical center, Bar ilan university, Israel
}

\section{Case report}

A 24 years old man was admitted to our department because of fever, diarrhea and fatigue. The patient, a Thai foreign worker had been well until ten days prior his admission when nausea, vomiting and watery diarrhea developed three days after eating raw pork. Two days prior to his admission a community physician prescribed him a symptomatic therapy that didn't help him recover, on the contrary, his condition escalated as he reported severe fatigue and muscular pain. He also had temperatures up to 39 along with chills.

On his emergency room admission, he looked sick, uncomfortable and weak, his temperature was 38.9, blood pressure $106 \backslash 57 \mathrm{mmHg}$ with tachycardia with HR of 108 per minute, respiration rate was 14 per minute. There was a maculopapular rash over his back, the remainder of the examination was normal. As for the laboratory tests, complete blood count revealed leukocytosis due to neutrophilia (12000) and severe eosinophilia (2230). Measurements of serum electrolytes and renal function has revealed severe hyponatremia (125), $\mathrm{CPK}=810$ and a positive Troponin of $0.245 \mathrm{nl}$, renal function and other electrolytes were in normal range. He was given NACL $0.9 \%$ saline and a course of Ceftriaxone 1grlday. An Electocardiogram was done due to the elevation of troponin values showing a diffuse elevation of ST segment in leads V1-V6 with normal voltage. Chest X-ray was normal, heart silhouette wasn't enlarged with clear costo-diaphragmatic recess. On the admission to our department, complete laboratory tests were done (Table 1). Because of the anamnestic information we suspected an infection of trichinosis, so he was put on albendazole $400 \mathrm{mg}$ twice a day and prednisone $30 \mathrm{mg}$ twice a day. Specimens of blood and stool were cultured and serology for EBV, CMV, HCV, HBV and HAV, HIV and blood cultures were sent, all were negative [1].

During the next days, the symptoms did not improve. The patient reported a cough and increasing muscle aches, his temperature had been falling and rising up to 39.8 , blood pressure was $90 \backslash 60$, he was still in tachycardia of about 110 per minute and his respiratory rate was 28 per minute. On his examinations he was unable to rise from bed because of severe muscle weakness and pain, crackles were heard over his lungs and leg edema $(+2)$ developed. On his daily laboratory blood test and blood gases, hemoglobin dropped $3.3 \mathrm{gr}$ down to $11.6 \mathrm{~g} / \mathrm{dL}$ and eosinophilia dropped to $1.3^{\star} 10 \mathrm{e} 3 / \mathrm{uL}$, troponin was negative and liver transaminases and muscular markers such as CPK were worsened (Table 1) On arterial blood gases (ABG) respiratory alkalosis with fully metabolic compensation was shown. We ordered another chest $\mathrm{X}$-ray revealing a pleural effusion on the right costo diaphragmatic recess. An ultrasound of the abdomen was normal, transthoracic echocardiography revealed a pericardial effusion. In summary this patient was demonstrating a suspected parasitic infection with hepatitis, myopericarditis and acute inflammatory anemia. Over his hospital stay we received a positive anti-Trichinella Antibody +++.

\section{Discussion}

Trichinenosis also called trichinellosis is a parasitic infection caused by nematode helminthes of trichinella. This disease is a zoonosis that could be acquired when consuming of undercooked or raw meat or meat infected with trichinella larvae. The most common etiology is consumption of pork, but meat of other animals may cause the disease as well.

Most of the individuals infected with this parasite experience mild GI symptoms like diarrhea and nausea related to penetration of the larvae of the intestinal mucosa. This manifestation known as the enteric phase are in most cases related to upper GI symptoms, our patient complained about upper abdominal pain, vomiting, nausea and watery diarrhea and fever which haven't resolved for 7 days.

Generally, the enteral phase is present through the first 2-6 weeks of the infection, though the symptoms start to abate. At this time, as in the case of our patient- new signs and symptoms develop due to the next stage of the disease, Migratory stage, in which larvae develop in muscles. In this stage the symptoms is related to the amount of consumed larvae, so mild to moderate amount of infection can produce the following: myalgia, headache, skin rash, fever, paralysis like state and facial edema.

Our patient surprisingly, reported only few of these, he developed myalgia, fatigue, and shivering. In his physical examination we reported a maculopapular rash and extreme myositis. Accordingly, and with correlation with the nature of this infection, leukocytosis with predominance of eosinophils developed. Eosinophilia is the most characteristic and earliest laboratory finding of this infection, and it is believed to be correlated with the degree of myalgia. In this acute phase a decrease in eosinophil levels in severe trichinella infection is sign of bad prognosis.

Despite all signs, symptoms and laboratory finding so far indicating a trichinella disease, the fact that this patient also developed hepatitis myopercarditis would rise doubts to our diagnosis. In a retrospective analysis over 154 patients who were identified with trichenillosis cases, only 2 patients ( $1.3 \%)$ were identified as cases of myopericarditis.

Myocarditis was diagnosed according to clinical symptoms and high cardiac markers such as troponin and unspecific ECG changes which resolved in 4 days, and pericarditis was suspected due to complains about dyspnea palliated by leaning and indeed when performed an echocardiography, it exposed pericardial effusion.

Correspondence to: Assy N, Department of Internal Medicine A, Galilee medical center, Bar ilan university, Israe; E-mail: NimerA@gmc.gov.il

Received: September 03, 2016; Accepted: September 20, 2016; Published: September 24, 2016 
Furthermore hypotension was reported, in his physical examination JVP was negative and heart sounds were hyper dynamic.

In an overview of 17 epidemiological studies on trichinellosis (including 5268), cardiovascular events occurred in $26 \%$ of the patients trichinosis cardiovascular involvement. ECG changes were the most common finding (89\%). The diagnosis of cardiovascular involvement was based on cluster of symptoms signs and laboratory tests. On another research including 1039 cases of trichinella reported, 20\% of the patients had ecg changes of myocardial involvement.

On the other hand, bessoudo et al. revealed that pericardial effusions might occur in the absence of ecg changes.

In an analysis of cases reported trichinella infection, little was found about hepatic involvement especially hepatitis. On a metaanalysis of 32 cases reported, hepatomegaly was reported in $34.4 \%$, hypoalbuminnemia $40.6 \%$ and hypoprotienemia in $65.5 \%$.

The pathogenesis of the hepatic involvement is due to the penetration of the larvae to the hepatic sinusoids. This hepatic changes may be seen through or after the intestinal phase and could be attributed to direct injury ( the larvae) or indirect injury ( immunological or eosinophilia) and a lond course of the disease might result in irreversible damage.. Nevertheless hepatic injury does not represent a common feature in human trichinosis.

Though extremely rare jaundice and hepatomegaly on physical examination were clinical signs to liver injury, in our patient he didn't develop jaundice neither hepatomegaly. On the other hand the hypoalbuminemia he developed may be the result of acute inflammation or hepatocellular dysfunction, allergic capillaropathy and deficitary digestion and absorption of proteins due to alterations of the intestinal mucosa.

On an metanalysis which demonstrated a hepatic involvement in trichinilosis infection, 32 cases were thoroughly detailed to allow pooled analysis of hepatic involvement concluded that hepatic injury were mainly evidence in fatal cases of trichinilosis, which wasn't the case.

Though the metanalysis reports evidence of hepatitis like modification acoompanid by inflammatory infiltrates and hepatocellular dystrophy, none were reporting acute hepatitis.

As for our patient, he developed non-viral acute hepatitis (HIV, HAV, HBV, HCV, HDV, EBV and CMV were all excluded), his serum transaminases were both elevated.

These enzymes are released to the peripheral blood in greater amounts than their normal concentrations when there is a damage to the liver cell membrane resulting in higher permeability, same pathogenesis is believed to be caused by trichinilosis infection $[2,3]$.

In our patient reported, there was an evidence of modest increase of his transaminases, values were lower than 300 and a ratio of ALT: AST bigger than 1, indicating an infiltrative liver injury since ALT is found primarily in the liver, this pattern had not been reported so far in any trichinella infection on human being although 3 cases demonstrated an elevated transaminases but the specific number were not published.

To summarize, Trichinella infection is a multi-organ infiltrative disease despite the fact that little was reported in the recent years.

Hepatic and cardiologic complication were reported mostly in fatal cases of the disease, therefor wide laboratory tests and cardiac monitoring should be performed in patients demonstrating clinical features of the disease to minimize its mortality.

\section{References}

1. Bruno Gottstein, Edoardo Pozio and Karsten No“ckler (2009) Epidemiology, Diagnosis, Treatment, and Control of Trichinellosis. Clinical Microbiology Reviews: 127-145.

2. Buchanan TM, Faber LC, Feldman RA (1974) Brucellosis in the United States, 1960 1972. An abattoir-associated disease. Part I. Clinical features and therapy. Medicine (Baltimore) 53: 403-413. [Crossref]

3. Young EJ (1995) An overview of human brucellosis. Clin Infect Dis 21: 283-289. [Crossref]

Copyright: (C2016 Boulus M. This is an open-access article distributed under the terms of the Creative Commons Attribution License, which permits unrestricted use, distribution, and reproduction in any medium, provided the original author and source are credited. 